\title{
Comprehensive Evaluation on Employee Satisfaction of Mine Occupational Health and Safety Management System Based on Improved AHP and 2-Tuple Linguistic Information
}

\author{
Jiangdong Bao ${ }^{1}$, Jan Johansson ${ }^{1}$ and Jingdong Zhang ${ }^{2, *}$ \\ 1 Centre of Advanced Mining and Metallurgy, CAMM, Department of Human Work Science, \\ Luleå University of Technology, Luleå 97751, Sweden; bao.jiangdong@ltu.se (J.B.); \\ Jan.Johansson@ltu.se (J.J.) \\ 2 Research Center for Environment and Health, Zhongnan University of Economics and Law, \\ Wuhan 430000, China \\ * Correspondence: jdzhang@zuel.edu.cn; Tel.: +86-27-8838-6196
}

Academic Editor: Yongrok Choi

Received: 5 October 2016; Accepted: 12 January 2017; Published: 18 January 2017

\begin{abstract}
In order to comprehensively evaluate the employee satisfaction of mine occupational health and safety management system, an analytic method based on fuzzy analytic hierarchy process and 2-tuple linguistic model was established. Based on the establishment of 5 first-grade indicators and 20 second-grade ones, method of improved AHP and the time-ordered Weighted Averaging Operator (T-OWA) model is constructed. The results demonstrate that the employee satisfaction of the mine occupational health and safety management system is of the 'general' rank. The method including the evaluation of employee satisfaction and the quantitative analysis of language evaluation information ensures the authenticity of the language evaluation information.
\end{abstract}

Keywords: employee satisfaction; mine; OHSAS18001; improved AHP; 2-tuple linguistic information

\section{Introduction}

To a large extent, employee satisfaction not only determines the survival and development of enterprises, but also it is the core idea of quality, environment, occupational health, and safety management systems. At present, OHSAS18001 has protected the health and safety of employees and promote the development of economy to a certain extent in the mine system. Some common problems are still to be solved such as individual protection not being taken well, insufficient necessary training, operating specifications being ruled but hard to implement, safety culture not being well valued, improper handling of related party complaints, lack of awareness of environmental protection, etc. Aiming at the problems employees are most concerned about, individual development of employees, effectiveness of system operation, safety and health performance, safety culture, and environmental sustainable development are comprehensively evaluated to improve employee satisfaction and OHSAS18001 operation.

Scientific and effective evaluation of enterprise employee satisfaction is not only the requirement of the enterprise strategy development, but also it has an important reference value for the continuous improvement of the enterprise [1]. At present, the research on employee satisfaction mainly focuses on its composition, influencing factors, and evaluation systems. The evaluation method [1] concentrates upon the balance integral card, structural equation model, grey system model, fuzzy analytic hierarchy process, principal component analysis, factor analysis, logistic regression analysis, and so on. Normally, the methods of aggregation of language information include transforming linguistic information into fuzzy numbers to calculate the fuzzy numbers [2,3], and adopting the linguistic method to calculate the 
subscript of the language phrase [4]. However, these methods cannot match the results accurately to the initial language evaluation. However, the evaluation of employee satisfaction is a kind of subjective value judgment, which is fuzzy and uncertain. During the evaluation, it is usually difficult for the participants to accurately determine the degree of satisfaction with precise numbers or language, and the acquired language information is usually not directly involved in mathematical calculating, resulting in a loss of information and accuracy [5].

Herrera and Martínez [6] put forward 2-tuple linguistic information and the corresponding aggregation operator for the first time, which can solve the defects of the above methods, and can ensure the integrity and authenticity of the information in the process of language information gathering and processing. Therefore, this paper applies 2-tuple linguistic information processing method integrated with method of improved AHP which can ensure the reliability of the indicator weight. The feasibility and effectiveness of the method is verified by a case.

\section{Mine Occupational Health and Safety Management System Employee Satisfaction Indicators}

OHSAS18001 occupational health and safety is one of the advanced modernized production safety management methods widely carried out in the world. It focuses on systematic ideas of health and safety management. The aim of the establishment of a complete set of occupational health and safety mechanisms is to control and reduce the risk of occupational health and safety, to minimize the occurrence of production accidents and occupational disease and to provide an important reference for the coordination and sustainable development of human, economic, social, resource, and environmental protection.

Safety benefit is an effective evaluation method of OHSAS18001, which refers to realization of safety level, the benefits to society, the community, and the individual. Additionally, they are composed of the sum (decrease loss output and increment output) — total safety investment ratio [7]. The key point of calculating safety benefits is to calculate the safety output as shown below [7]: $B$ (safety output) $=B_{1}$ (decrease loss output) $+B_{2}$ (increment output). In the formula, $B_{1}$ (decrease loss output) $=\sum$ (decrease loss increment) = early loss (before safety measures)-later loss (after safety measures); $B_{2}$ (increment output $)=$ productivity contribution $\times$ GDP. According to the statistics of the financial department of the mining industry, the safety benefit from 2009 to 2010 was 958.321 and 923.672 10-thousand Yuan (without the introduction of OHSAS18001), while the safety benefit from 2011 to 2015 was 1231.514, $1472.079,1261.509,1805.606$, and 2136.734 10-thousand Yuan (with the introduction of OHSAS18001). It can be concluded that OHSAS18001 has promoted the development of the safety benefit.

In full consideration of the basis of employee satisfaction evaluation indicators given by domestic and foreign scholars [8-15], combined with the OHSAS18001 standard requirements [16], employee satisfaction evaluation first and second-grade indicators of the OHSAS18001 are given as is shown in Table 1.

Table 1. Occupational health and safety management system employee satisfaction indicators.

\begin{tabular}{ll}
\hline First-Grade Indicator & Second-Grade Indicator \\
\hline & $u_{11}$ Training opportunities \\
$U_{1}$ Individual development of OHSAS & $u_{12}$ People-oriented \\
& $u_{13}$ Information openness \\
& $u_{14}$ Access to development opportunities \\
\hline & $u_{21}$ Continuous improvement \\
& $u_{22}$ Communication \\
& $u_{23}$ Coordination and cooperation of work \\
& $u_{24}$ Effectiveness of management \\
\hline & $u_{31}$ Safety performance \\
& $u_{32}$ Health performance \\
$U_{3}$ Economical efficiency of OHSAS & $u_{33}$ Operating performance \\
& $u_{34}$ Economic structure \\
\hline
\end{tabular}


Table 1. Cont.

\begin{tabular}{ll}
\hline First-Grade Indicator & Second-Grade Indicator \\
\hline \multirow{3}{*}{$U_{4}$ Social efficiency of OHSAS } & $u_{41}$ Related party \\
& $u_{42}$ Safety culture \\
& $u_{43}$ Organizational performance and culture \\
& $u_{44}$ Social culture \\
\hline \multirow{2}{*}{$U_{5}$ Environmental efficiency of OHSAS } & $u_{51}$ Environment and safety \\
& $u_{52}$ Environment and health \\
& $u_{53}$ Ecological management cost \\
& $u_{54}$ Environmental sustainable development \\
\hline
\end{tabular}

\subsection{Individual Development of OHSAS}

Individual development of OHSAS is to meet the needs of people's all-round and sustainable development. Implementation of the project is bound to affect the people, mainly in the following aspects: training opportunities, people-oriented, information openness, and access to development opportunities.

\subsection{Effectiveness of OHSAS}

Effectiveness means the activity of planning and the degree to which the planning results are achieved. Effectiveness of OHSAS18001 means the consistency between the personnel arrangement, communication, and consultation; management; and the fulfillment of objectives. According to the requirements of OHSAS, effectiveness is mainly manifested in the following aspects: continuous improvement, communication, coordination and cooperation of work, and effectiveness of management.

\subsection{Economic Efficiency of OHSAS}

Economic efficiency means an economic activity that can be consumed by the least physical labor to achieve the greatest economic results and to coordinate sustainable development with society, the environment, and so on. Economic efficiency of OHSAS means the promotion of economic benefit of safety and health management on the enterprise during OHSAS18001 operation, which mainly manifests in the following aspects: safety performance, health performance, operating performance, and economic structure.

\subsection{Social Efficiency of OHSAS}

Social efficiency means a living creature as individuals of collective activities, or as a member of society with characteristics which are beneficial to collective activities and social sustainable development. Social efficiency of OHSAS18001 not only includes characteristics which is beneficial to the collective activities and social sustainable development, but it also includes physical and mental health and safety of employees engaged in production activities as well as employees' sense of safety, beliefs, culture, values, etc. It is mainly manifested in the following aspects: related party, safety culture, organizational performance, and culture and social culture.

\subsection{Environmental Efficiency of OHSAS}

Environmental efficiency is a kind of activities of adapting to nature and protecting the nature which is mainly manifested in the following aspects: environment and safety, environment and health, ecological management cost, and environmental sustainable development.

\section{Indicator Weight of Mine Occupational Health and Safety Management System Based on Improved AHP Model}

\subsection{Evaluation Weight Set}

In this paper, the importance among indicators is scored by the relevant experts, to determine the weight value of each indicator in this factor and to construct the judgment matrix referring to $1 \sim 9$ scale 
method proposed by Thomas L. Saaty [17] to determine the specific values. If the parameter on the horizontal axis of each line was less important than the parameter on the vertical axis of the column, it carried a value between 1 and 9. Oppositely, it carried the value between the reciprocals of $1 / 2$ and 1/9 [18]. Jian Shi et al. [19] pointed out that the '1-9' scales method of AHP by T.L. Saaty was used construct the comparison matrix whose consistent effect was insufficient. The traditional AHP method was thought to be only for specific qualitative indicators. On the other hand, the issues with both qualitative and quantitative indicators are not discussed enough [20]. The traditional AHP method talked more about the consistency of judgment matrix than the rationality of judgment matrix [21-23]. Shuang Chen et al. [24] preliminarily applied the improved '9/9-9/1' AHP method to weight sorting. Jiangdong Bao et al. [25] applied the improved AHP together with FMEA (Failure Mode and Effects Analysis) to assess the occupational health in a mining industry. In this paper, the improved AHP method integrated with 2-tuple linguistic information is used to evaluate the employee satisfaction of mine occupational health and safety management system to provide a more scientific and accurate decision basis.

The traditional AHP method has some shortcomings about the experts scoring as shown follows:

- $\quad$ The ' $1-9$ ' scales method would make the accuracy rate low.

- The method would make the connection of levels confused.

- The method would make data processing cumbersome.

The method is optimized and improved with a new ‘ $9 / 9-9 / 1^{\prime}$ scale as shown in Table 2.

Table 2. The experts scoring table of the importance among indicators of the traditional and new scale.

\begin{tabular}{ccc}
\hline 9/9-9/1 (Intensity of Importance) & 1-9 (Intensity of Importance) & Definition \\
\hline $9 / 9$ & 1 & Equal importance \\
$9 / 8$ & 2 & Equal to moderate importance \\
$9 / 7$ & 3 & Moderate importance \\
$9 / 6$ & 4 & Moderate to strong importance \\
$9 / 5$ & 5 & Strong importance \\
$9 / 4$ & 6 & Strong to very strong importance \\
$9 / 3$ & 7 & Very strong importance \\
$9 / 2$ & 8 & Very to extremely strongly importance \\
$9 / 1$ & 9 & Extreme importance \\
Reciprocals & & The judgement of parameter $i$ compared \\
& & with parameter $j$ is $a_{i j}$, then the one of \\
parameter $j$ compared with parameter $i$ is $a_{j i}$
\end{tabular}

\subsection{Consistency Checking}

The test index for the consistency of judgment is as follows: $C R=C I / R I$; In the equation, $C I=(\lambda-n) /(n-1), n$ is the order and $\lambda$ is the maximal eigenvalue of the judgment matrix. $R I$ is random consistency index of judgment matrix [26]. Suppose the set $R I$ is shown in Table 3.

If $C R \leq 10 \%$, the matrix is consistent and AHP can be continued. If $C R>10 \%$, it requires revision because the matrix is not consistent. In this paper, the root mean square method is used to carry out the consistency test. The model calculation procedure is as follows:

(1) Multiply the judgments of indicators by line $u_{i j}=\prod_{j=1}^{n} b_{i j}$

(2) The $n$th root of the resultant product $u_{i}=\sqrt[n]{u_{i j}}$

(3) Normalize the root mean square vector and get the feature vector $w_{i}=\frac{u_{i}}{\sum_{i=1}^{n} u_{i}}$ 
(4) Calculate the largest eigenvalue [27,28] of the judgment matrix $\lambda_{\max }=\sum_{i=1}^{n} \frac{(A W)_{i}}{(n W)_{i}}$, where $A W$ means the parameter on the horizontal axis multiplied by the matrix of the weight.

(5) Calculate $C R=C I / R I=(\lambda-n) /(n-1) / R I$, where $R I$ set value is shown as follows.

Table 3. RI set value.

\begin{tabular}{ccccccccc}
\hline $\boldsymbol{n}$ & $\mathbf{1}$ & $\mathbf{2}$ & $\mathbf{3}$ & $\mathbf{4}$ & $\mathbf{5}$ & $\mathbf{6}$ & $\mathbf{7}$ & $\mathbf{8}$ \\
\hline$R I$ & 0 & 0 & 0.58 & 0.9 & 1.12 & 1.24 & 1.32 & 1.41 \\
\hline
\end{tabular}

\section{The Employee Satisfaction Model of Mine Occupational Health and Safety Management System Based on 2-Tuple Linguistic Information}

\subsection{2-Tuple Linguistic Information}

Professor Herrera, a Spanish scholar, put forward 2-tuple linguistic information [6] method of the linguistic aggregation information for the first time in 2000. It can solve the problem of the loss and distortion of the language information so as to make the evaluation information more accurate. At the same time, he also proposed Time-ordered Weighted Averaging Operator (T-OWA) based on 2-tuple linguistic information in 2001. It is successfully applied to multi attribute evaluation and decision analysis of multi granularity linguistic scales [29].

2-Tuple linguistic information is the result of language evaluation with $\left(s_{k}, u_{k}\right)$, in which $s_{k}$ is the first $K$ elements of the language information evaluation set $S$, and $u_{k}$ is the symbol conversion value with $u_{k} \in[-0.5,0.5)$. It represents the deviation between the linguistic information set and the most appropriate linguistic phrase in the pre-defined language information set $S . s_{k}$ and $u_{k}$ are described as follows.

Definition 1 [29,30]. Language information evaluation set $S, S=\left\{s_{1}=W\right.$ (worse), $s_{2}=B$ (bad), $s_{3}=N$ (normal) $s_{4}=G($ good $), s_{5}=E$ (excellent) $s_{6}=V G($ very good $\left.)\right\}$.

Definition $2[29,30]$. Let $A=\left\{a_{1}, a_{2}, \ldots, a_{m}\right\}$ be a set of linguistic terms to be aggregated, the convex combination is defined in a recursive way as the following.

For $m=2$

$$
C^{2}\left\{\left\{w_{1}, 1-w_{1}\right\},\left\{b_{1}, b_{2}\right\}\right\}=\left(w_{1} \odot s_{j}\right) \oplus\left(\left(1-w_{1}\right) \odot s_{i}\right)=s_{k}, s_{j}, s_{i} \in S
$$

Such that $k=\min \left\{g, i+\operatorname{round}\left(w_{1} \cdot(j-i)\right)\right\}$ where $g+1$ is the cardinality of $S$, round (.) is the usual round operation, and $b_{1}=s_{j}, b_{2}=s_{i}$.

For $m>2$

$$
\begin{gathered}
C^{m}\left\{w_{k}, b_{k}, k=1, \ldots, m\right\}=\left(w_{1} \odot b_{1}\right) \oplus\left(\left(1-w_{1}\right)\right) \odot C^{m-1}\left\{r / h, b_{h}, h=2, \ldots, m\right\} \\
C^{2}\left\{\left\{w_{1}, 1-w_{1}\right\} b_{1}, C^{m-1}\left\{r / h, b_{h}, h=2, \ldots, m\right\}\right\}
\end{gathered}
$$

where $W=\left[w_{1}, \ldots, w_{m}\right]$ is a weighting vector associated with $\mathrm{A}$, such that, (i) $w_{i} \in[0,1]$; and (ii) $\Sigma_{i} w_{i}=1$; and $B=\left\{b_{1}, \ldots, b_{m}\right\}$ is a vector such that $B=\left\{a_{\sigma(1)}, \ldots, a_{\sigma(m)}\right\}$, where $a_{\sigma(j)} \leq a_{\sigma(i)} \forall_{i} \leq j$, with $\sigma$ being a permutation over the values $a_{i} \times r / h=w_{h} / \Sigma_{2}^{m} w_{k}, h=2, \ldots, m$. 
Definition $3[29,30]$. Let $\beta$ be the result of an aggregation of the indexes of a set of labels assessed in a linguistic term set $S$, i.e., the result of a symbolic aggregation operation. $\beta \in[0, g]$, being $g+1$ the cardinality of $S$. Let $i=\operatorname{round}(\beta)$ and $\alpha=\beta-i$ be two values such that $i \in[0, g]$ and $\alpha \in[-0.5,0.5)$ then $\alpha$ is called $a$ symbolic translation.

Definition $4[29,30]$. If the real number $\beta \in[0, g]$ is the real one of the linguistic evaluation set $S$, then the $\beta$ can be obtained by the function $\Delta$ and $\Delta^{-1}$ to achieve the basic conversion of 2-tuple linguistic information. $\Delta(\beta)=\left(s_{k}, a_{k}\right), k=\operatorname{Round}(\beta)$. Round means to round up and round down number operator. $a_{k}=\beta-k ; \Delta^{-1}\left(s_{k}, a_{k}\right)=k+a_{k}=\beta$.

Definition $5[29,30]$. If $\left(s_{k}, a_{k}\right)$ and $\left(s_{m}, a_{m}\right)$ are the two 2-tuple linguistic information, the comparison operators of the two 2-tuple linguistic operators have two kinds of cases. If $k<m$, then $\left(s_{k}, a_{k}\right)<\left(s_{m}, a_{m}\right)$; if $k=m$, then there are three kinds of situations: (1) If $a_{k}=a_{m}$, then $\left(s_{k}, a_{k}\right)=\left(s_{m}, a_{m}\right)$; (2) If $a_{k}>a_{m}$, then $\left(s_{k}, a_{k}\right)>\left(s_{m}, a_{m}\right)$; (3) If $a_{k}<a_{m}$, then $\left(s_{k}, a_{k}\right)<\left(s_{m}, a_{m}\right)$.

Example 1 [6]. Let us suppose a symbolic aggregation operation over labels assessed in $S=\left\{s_{0}, s_{1}, s_{2}, s_{3}, s_{4}, s_{5}, s_{6}\right\}$ that obtains as its result $\beta=2.8$, then the representation of this counting of information by means of a 2-tuple will be $\Delta(2.8)=\left(s_{3},-0.2\right)$. Graphically, it is represented in Figure 1.

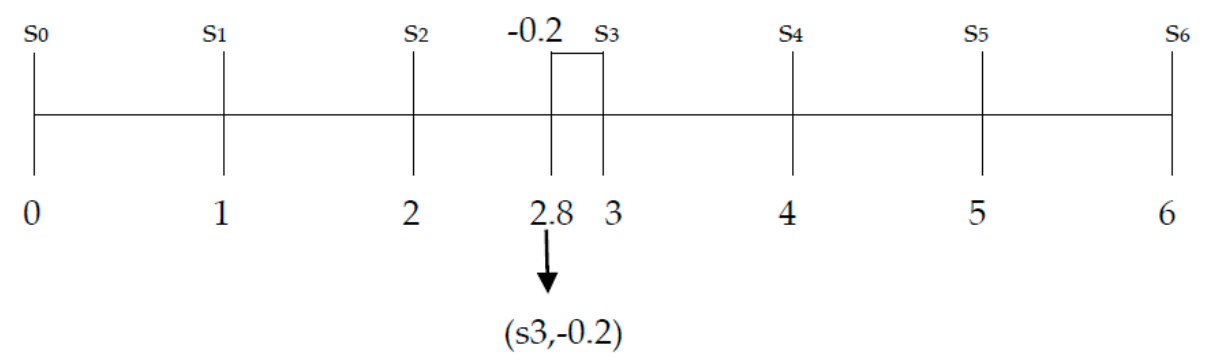

Figure 1. Example of a symbolic translation computation.

\subsection{The T-OWA Operator}

It is used to aggregate the linguistic evaluation information of the experts. The definition of T-OWA operator [30,31] is: If $\left\{\left(s_{1}, a_{1}\right),\left(s_{2}, a_{2}\right), \ldots,\left(s_{m}, a_{m}\right)\right\}$ is a set of 2-tuple linguistic evaluation information, and the definition of T-OWA operator $\Phi$ is defined as follows:

$$
(\bar{s}, \bar{a})=\Phi\left(\left(s_{1}, a_{1}\right),\left(s_{2}, a_{2}\right), \ldots,\left(s_{m}, a_{m}\right)\right)=\Delta\left(\sum_{i=1}^{m} c_{i} v_{i}\right), \bar{s} \in S, \bar{a} \in[-0.5,0.5)
$$

In the Equation (1), element $c_{i}$ of vector $C=\left[c_{1}, c_{2}, \ldots, c_{m}\right]$ represents the one of the first $i$ bit in the set $\left\{\Delta^{-1}\left(s_{i}, a_{i}\right), i=1,2, \ldots, m\right\}$, which is in accordance with the order of large to small. $c$ represents the weight vector of each expert.

\subsection{The Definition of Fuzzy Operator $Q(r)$}

The definition is as follows

$$
v_{i}=Q(i / m)-Q((i-1) / m), v_{i} \in[0,1], \sum_{i=1}^{m} v_{i}=1
$$




$$
Q\left(r_{i}\right)=\left\{\begin{array}{lc}
0 & r_{i}<a \\
\left(r_{i}-a\right) /(b-a) & a \leq r_{i} \leq b \\
1 & r_{i}>b
\end{array}\right.
$$

In the Equation (3), $a, b \in[0,1]$, and under such a principle of at least half, most, and as many as possible situation, parameter $(a, b)$ is $(0,0.5),(0.3,0.8),(0.5,1)$. Normally, the principle of most situation $(0.3,0.8)$ is used to calculate the information. That is, $a=0.3$ and $b=0.8$, and $r_{i}=i / m$, where $i=1,2, \ldots, m ; m=$ number of experts.

\subsection{2-Tuple Linguistic Information after Integration of the Second Grade Indictor}

2-Tuple linguistic information after integration of the second grade indictor can be got by the formula:

$$
\left(s_{j}, a_{j}\right)=\Delta\left(\sum_{k=1}^{l} w_{j k}\right) \Delta^{-1}\left(s_{k}, a_{k}\right)
$$

where $w_{j k}$ is the $k$ th weight of second grade indicator of the $j$ th first indicator, and $j=1,2, \ldots, q ; k=1$, $2, \ldots, l$.

\subsection{Comprehensive 2-Tuple Linguistic Information of First Grade Indicator}

Comprehensive 2-tuple linguistic information of the first grade indicator can be got by the formula:

$$
(s, a)=\Delta\left(\sum_{j=1}^{q} w_{j}\right) \Delta^{-1}\left(s_{j}, a_{j}\right), s \in S, a \in[-0.5,0.5)
$$

where $w$ is the weight of first grade indicator, thus the result of comprehensive 2-tuple linguistic information integrated with improved AHP method is obtained to evaluate the employee satisfaction of the mine OHSAS18001.

\section{Case Study}

The mine is located in the southwest of the Hubei Province in the central part of China, and it has general hydrogeological conditions. The mine lies in a subtropical monsoon climate zone with monsoon climate and abundant rainfall. The ore water unit boundary of this mine has a good water separation effect with marble at the bottom, good developed karst, and confined aquifers. Ground cracking and settlement collapse are the most important geological hazards in the mining area. The mine adopted a sublevel caving method integrated with advanced lifting equipment and efficient mining equipment with an annual output of three million tons of iron ore. Additionally, the iron concentrate grade achieves national advanced level of $66.5 \%$.

Additionally, OHSAS18001 has been utilized for the site for more than three years with a good reputation in the society as well as the local community. Utilizing the mine as an example, this paper evaluates and analyzes the employee satisfactory of OHSAS through an analytic hierarchy process (AHP) method and a 2-tuple linguistic evaluation information.

\subsection{The Indicator Weight and Consistency Test}

In order to ensure the validity and consistency of the evaluation model, the indicators need to be tested. Additionally, 30 Chinese senior staff with more than five years of working experience in the mine were invited to judge the importance of the indicators in the improved AHP method. To ensure the accuracy of judgment, the selected senior staff should be given appropriate training of the method which is also the requirement of random sampling. In this paper, the root mean square method is used to carry out the consistency test. The calculation procedure of the first grade indicators weight is shown as follows. 
According to the aforementioned formulas, calculation of first-grade indicator is obtained as shown in Table 4: $u_{i j}=\prod_{j=1}^{n} b_{i j}=1.831,1.000,0.778,1.143,0.615 ; u_{i}=\sqrt[n]{u_{i j}}=1.129,1.000,0.951$, 1.027, 0.907, where 5.014 means the sum of $u_{i} ; w_{i}=\frac{u_{i}}{\sum_{i=1}^{n} u_{i}}=0.225,0.199,0.190,0.205,0.181$, where 1.000 means the sum of $W_{i} ; \lambda_{\max }=\sum_{i=1}^{n} \frac{(A W)_{i}}{(n W)_{i}}=5.015$, where 5.015 means the average of $A W_{i} / W_{i}$; $C R=C I / R I=(\lambda-n) /(n-1) / 1.24=0.003<0.1$, Thus, the result has passed the consistency test.

Table 4. Calculation results of first-grade indicator.

\begin{tabular}{ccccccccccc}
\hline & $\boldsymbol{U}_{\mathbf{1}}$ & $\boldsymbol{U}_{\mathbf{2}}$ & $\boldsymbol{U}_{\mathbf{3}}$ & $\boldsymbol{U}_{\mathbf{4}}$ & $\boldsymbol{U}_{\mathbf{5}}$ & $\boldsymbol{u}_{\boldsymbol{i j}}$ & $\boldsymbol{u}_{\boldsymbol{i}}$ & $\boldsymbol{W}_{\boldsymbol{i}}$ & $A \boldsymbol{W}_{\boldsymbol{i}}$ & $A \boldsymbol{W}_{\boldsymbol{i}} / \boldsymbol{W}_{\boldsymbol{i}}$ \\
\hline$U_{1}$ & $9 / 9$ & $9 / 8$ & $9 / 8$ & $9 / 8$ & $9 / 7$ & 1.831 & 1.129 & 0.225 & 1.126 & 5.002 \\
$U_{2}$ & $8 / 9$ & $9 / 9$ & $9 / 9$ & $9 / 8$ & $9 / 9$ & 1.000 & 1.000 & 0.199 & 1.001 & 5.017 \\
$U_{3}$ & $8 / 9$ & $9 / 9$ & $9 / 9$ & $7 / 9$ & $9 / 8$ & 0.778 & 0.951 & 0.190 & 0.952 & 5.020 \\
$U_{4}$ & $8 / 9$ & $8 / 9$ & $9 / 7$ & $9 / 9$ & $9 / 8$ & 1.143 & 1.027 & 0.205 & 1.030 & 5.026 \\
$U_{5}$ & $7 / 9$ & $9 / 9$ & $8 / 9$ & $8 / 9$ & $9 / 9$ & 0.615 & 0.907 & 0.181 & 0.906 & 5.008 \\
& & & & & & & 5.014 & 1.000 & & 5.015 \\
\hline
\end{tabular}

In the same way, the weight of the second grade indicators can be obtained, and the results are shown as below:

$$
\begin{aligned}
& W_{u_{11}-u_{14}}=[0.236,0.458,0.131,0.175] ; W_{u_{21}-u_{24}}=[0.186,0.323,0.224,0.267] ; \\
& W_{u_{31}-u_{34}}=[0.215,0.249,0.224,0.312] ; W_{u_{41}-u_{44}}=[0.153,0.322,0.224,0.301] ; \\
& W_{u_{51}-u_{54}}=[0.349,0.152,0.198,0.301] ; W_{u_{1}-u_{5}}=[0.225,0.199,0.190,0.205,0.181] .
\end{aligned}
$$

\subsection{Semantic Comments on the Employee Satisfaction Evaluation Indicator}

According to the evaluation of 2-tuple linguistic information, the evaluation indicator is divided into six grades, as shown in Table 5.

Table 5. Grading instruction.

\begin{tabular}{cccc}
\hline Semantic Identity & Semantic Grade & Assessment Score & Grading Instruction \\
\hline$s_{1}$ & $W$ & 50 below & worse \\
$s_{2}$ & $B$ & $50-60$ & bad \\
$s_{3}$ & $N$ & $60-70$ & normal \\
$s_{4}$ & $G$ & $70-80$ & good \\
$s_{5}$ & $E$ & $80-90$ & excellent \\
$s_{6}$ & $V G$ & $90-100$ & very good \\
\hline
\end{tabular}

\subsection{Employee Satisfaction Evaluation Indicator Score and Corresponding 2-Tuple Linguistic Judgment Matrix}

Five senior employees $e_{1}, e_{2}, e_{3}, e_{4}$, and $e_{5}$ are randomly selected to comprehensively evaluate the semantic grade of Table 6 . 
Table 6. Indicator score and 2-tuple linguistic judgment matrix.

\begin{tabular}{ccccccc}
\hline $\begin{array}{c}\text { First-Grade } \\
\text { Indicator }\end{array}$ & $\begin{array}{c}\text { Second-Grade } \\
\text { Indicator }\end{array}$ & $\boldsymbol{e}_{\mathbf{1}}$ & $\boldsymbol{e}_{\mathbf{2}}$ & $\boldsymbol{e}_{3}$ & $\boldsymbol{e}_{4}$ & $\boldsymbol{e}_{5}$ \\
\hline \multirow{4}{*}{$U_{1}$} & $u_{11}$ & $E /\left(s_{5}, 0\right)$ & $G /\left(s_{4}, 0\right)$ & $N /\left(s_{3}, 0\right)$ & $G /\left(s_{4}, 0\right)$ & $N /\left(s_{3}, 0\right)$ \\
& $u_{12}$ & $N /\left(s_{3}, 0\right)$ & $N /\left(s_{3}, 0\right)$ & $G /\left(s_{4}, 0\right)$ & $N /\left(s_{3}, 0\right)$ & $G /\left(s_{4}, 0\right)$ \\
& $u_{13}$ & $G /\left(s_{4}, 0\right)$ & $E /\left(s_{5}, 0\right)$ & $G /\left(s_{4}, 0\right)$ & $E /\left(s_{5}, 0\right)$ & $N /\left(s_{3}, 0\right)$ \\
& $u_{14}$ & $N /\left(s_{3}, 0\right)$ & $E /\left(s_{5}, 0\right)$ & $G /\left(s_{4}, 0\right)$ & $N /\left(s_{3}, 0\right)$ & $G /\left(s_{4}, 0\right)$ \\
& $u_{21}$ & $N /\left(s_{3}, 0\right)$ & $N /\left(s_{3}, 0\right)$ & $G /\left(s_{4}, 0\right)$ & $B /\left(s_{2}, 0\right)$ & $B /\left(s_{2}, 0\right)$ \\
& $U_{22}$ & $G /\left(s_{4}, 0\right)$ & $E /\left(s_{5}, 0\right)$ & $G /\left(s_{4}, 0\right)$ & $G /\left(s_{4}, 0\right)$ & $E /\left(s_{5}, 0\right)$ \\
& $u_{23}$ & $E /\left(s_{5}, 0\right)$ & $G /\left(s_{4}, 0\right)$ & $G /\left(s_{4}, 0\right)$ & $E /\left(s_{5}, 0\right)$ & $G /\left(s_{4}, 0\right)$ \\
& $u_{24}$ & $G /\left(s_{4}, 0\right)$ & $G /\left(s_{4}, 0\right)$ & $N /\left(s_{3}, 0\right)$ & $G /\left(s_{4}, 0\right)$ & $E /\left(s_{5}, 0\right)$ \\
& $u_{31}$ & $N /\left(s_{3}, 0\right)$ & $G /\left(s_{4}, 0\right)$ & $E /\left(s_{5}, 0\right)$ & $G /\left(s_{4}, 0\right)$ & $E /\left(s_{5}, 0\right)$ \\
& $u_{32}$ & $E /\left(s_{5}, 0\right)$ & $E /\left(s_{5}, 0\right)$ & $G /\left(s_{4}, 0\right)$ & $E /\left(s_{5}, 0\right)$ & $G /\left(s_{4}, 0\right)$ \\
& $u_{33}$ & $N /\left(s_{3}, 0\right)$ & $G /\left(s_{4}, 0\right)$ & $N /\left(s_{3}, 0\right)$ & $E /\left(s_{5}, 0\right)$ & $E /\left(s_{5}, 0\right)$ \\
& $u_{34}$ & $G /\left(s_{4}, 0\right)$ & $N /\left(s_{3}, 0\right)$ & $N /\left(s_{3}, 0\right)$ & $E /\left(s_{5}, 0\right)$ & $E /\left(s_{5}, 0\right)$ \\
$U_{4}$ & $u_{41}$ & $G /\left(s_{4}, 0\right)$ & $E /\left(s_{5}, 0\right)$ & $G /\left(s_{4}, 0\right)$ & $G /\left(s_{4}, 0\right)$ & $G /\left(s_{4}, 0\right)$ \\
& $u_{42}$ & $E /\left(s_{5}, 0\right)$ & $N /\left(s_{3}, 0\right)$ & $N /\left(s_{3}, 0\right)$ & $G /\left(s_{4}, 0\right)$ & $E /\left(s_{5}, 0\right)$ \\
& $u_{43}$ & $G /\left(s_{4}, 0\right)$ & $G /\left(s_{4}, 0\right)$ & $E /\left(s_{5}, 0\right)$ & $N /\left(s_{3}, 0\right)$ & $G /\left(s_{4}, 0\right)$ \\
& $u_{44}$ & $N /\left(s_{3}, 0\right)$ & $G /\left(s_{4}, 0\right)$ & $N /\left(s_{3}, 0\right)$ & $G /\left(s_{4}, 0\right)$ & $E /\left(s_{5}, 0\right)$ \\
$U_{5}$ & $u_{51}$ & $G /\left(s_{4}, 0\right)$ & $N /\left(s_{3}, 0\right)$ & $E /\left(s_{5}, 0\right)$ & $G /\left(s_{4}, 0\right)$ & $N /\left(s_{3}, 0\right)$ \\
& $u_{52}$ & $G /\left(s_{4}, 0\right)$ & $N /\left(s_{3}, 0\right)$ & $N /\left(s_{3}, 0\right)$ & $G /\left(s_{4}, 0\right)$ & $N /\left(s_{3}, 0\right)$ \\
& $u_{53}$ & $N /\left(s_{3}, 0\right)$ & $N /\left(s_{3}, 0\right)$ & $N /\left(s_{3}, 0\right)$ & $E /\left(s_{5}, 0\right)$ & $N /\left(s_{3}, 0\right)$ \\
& $u_{54}$ & $G /\left(s_{4}, 0\right)$ & $E /\left(s_{5}, 0\right)$ & $G /\left(s_{4}, 0\right)$ & $G /\left(s_{4}, 0\right)$ & $N /\left(s_{3}, 0\right)$ \\
\hline
\end{tabular}

5.4. The Weight Vector and 2-Tuple Linguistic Information of the Second Grade Indicator

According to Formulas (2) and (3), the weight of the score is obtained as follows: $V=[0,1 / 5,2 / 5,2 / 5,0]^{T}$; According to Definition 3, the vector of $u_{11}$ in accordance with the order from large to small is calculated as follows: $C=[5,4,3,3,2]$; According to Formula (1), it can be calculated as follows: $(\bar{s}, \bar{a})=\Phi\left(\left(s_{1}, a_{1}\right),\left(s_{2}, a_{2}\right), \ldots\left(s_{5}, a_{5}\right)\right)=\Delta\left(\sum_{i=1}^{5}\right)=3.100$, According to Definition 3 , the 2-tuple linguistic information after the aggregation of $u_{11}$ is obtained as follows: $(s 3,0.100)$; In the same way, the 2-tuple linguistic information after the aggregation of other second grade indicators can be obtained as in Table 7. According to the Formula (4), the 2-tuple linguistic information after integration of the second grade indictor as follows:

$$
\begin{aligned}
& \left(s_{1}, a_{1}\right)=\Delta\left(\sum_{k=1}^{4} w_{1 k} \Delta^{-1}\left(s_{k}, a_{k}\right)\right)=\Delta\left(\sum_{k=1}^{4} w_{1 k}\left(k+a_{k}\right)\right) \\
& =\Delta((0.236 \times 3.100)+(0.458 \times 3.200)+(0.131 \times 3.800)+(0.175 \times 3.600) \\
& =\Delta(3.330)=\left(s_{3}, 0.330\right)
\end{aligned}
$$

In the same way, the comprehensive $\beta$ value and the comprehensive 2-tuple linguistic information can be obtained as shown in Table 7 . 
Table 7. Indicator weight $\beta$ value and 2-tuple linguistic information.

\begin{tabular}{|c|c|c|c|c|c|c|c|}
\hline $\begin{array}{l}\text { First-Grade } \\
\text { Indicator }\end{array}$ & Weight & $\begin{array}{l}\text { Second-Grade } \\
\text { Indicator }\end{array}$ & Weight & $\begin{array}{l}\beta \text { Value of Second } \\
\text { Grade Indicator }\end{array}$ & $\begin{array}{l}\text { 2-Tuple Linguistic Information } \\
\text { of Second-Grade Indicator }\end{array}$ & $\begin{array}{l}\text { Comprehensive } \beta \text { Value of } \\
\text { Second-Grade Indicator }\end{array}$ & $\begin{array}{l}\text { Comprehensive 2-Tuple } \\
\text { Linguistic Information of } \\
\text { Second-Grade Indicator }\end{array}$ \\
\hline \multirow{4}{*}{$u_{1}$} & \multirow{4}{*}{0.292} & $u_{11}$ & 0.236 & 3.100 & $\left(s_{3}, 0.100\right)$ & \multirow{4}{*}{3.330} & \multirow{4}{*}{$\left(s_{3}, 0.330\right)$} \\
\hline & & $u_{12}$ & 0.458 & 3.200 & $\left(s_{3}, 0.200\right)$ & & \\
\hline & & $u_{13}$ & 0.131 & 3.800 & $\left(s_{4},-0.200\right)$ & & \\
\hline & & $u_{14}$ & 0.175 & 3.600 & $\left(s_{4},-0.400\right)$ & & \\
\hline \multirow{4}{*}{$u_{2}$} & \multirow{4}{*}{0.204} & $u_{21}$ & 0.186 & 2.600 & $\left(s_{3},-0.400\right)$ & \multirow{4}{*}{3.740} & \multirow{4}{*}{$\left(s_{4},-0.260\right)$} \\
\hline & & $u_{22}$ & 0.323 & 4.200 & $\left(s_{4}, 0.200\right)$ & & \\
\hline & & $u_{23}$ & 0.224 & 4.200 & $\left(s_{4}, 0.200\right)$ & & \\
\hline & & $u_{24}$ & 0.267 & 3.600 & $\left(s_{4},-0.400\right)$ & & \\
\hline \multirow{4}{*}{$u_{3}$} & \multirow{4}{*}{0.188} & $u_{31}$ & 0.215 & 4.200 & $\left(s_{4}, 0.200\right)$ & \multirow{4}{*}{4.090} & \multirow{4}{*}{$\left(s_{4}, 0.090\right)$} \\
\hline & & $u_{32}$ & 0.249 & 4.600 & $\left(s_{5},-0.400\right)$ & & \\
\hline & & $u_{33}$ & 0.224 & 3.800 & $\left(s_{4},-0.200\right)$ & & \\
\hline & & $u_{34}$ & 0.312 & 3.800 & $\left(s_{4},-0.200\right)$ & & \\
\hline \multirow{4}{*}{$u_{4}$} & \multirow{4}{*}{0.200} & $u_{41}$ & 0.153 & 3.600 & $\left(s_{4},-0.400\right)$ & \multirow{4}{*}{3.750} & \multirow{4}{*}{$\left(s_{4},-0.250\right)$} \\
\hline & & $u_{42}$ & 0.322 & 3.800 & $\left(s_{4},-0.200\right)$ & & \\
\hline & & $u_{43}$ & 0.224 & 4.000 & $\left(s_{4}, 0.000\right)$ & & \\
\hline & & $u_{44}$ & 0.301 & 3.600 & $\left(s_{4},-0.400\right)$ & & \\
\hline \multirow{4}{*}{$u_{5}$} & \multirow{4}{*}{0.115} & $u_{51}$ & 0.349 & 3.600 & $\left(s_{4},-0.400\right)$ & \multirow{4}{*}{3.330} & \multirow{4}{*}{$\left(s_{3}, 0.330\right)$} \\
\hline & & $u_{52}$ & 0.152 & 2.600 & $\left(s_{3},-0.400\right)$ & & \\
\hline & & $u_{53}$ & 0.198 & 3.000 & $\left(s_{3}, 0.000\right)$ & & \\
\hline & & $u_{54}$ & 0.301 & 3.600 & $\left(s_{4},-0.400\right)$ & & \\
\hline
\end{tabular}


In the same way, comprehensive 2-tuple linguistic information of the first grade indicator can be calculated as follows:

$$
\begin{aligned}
& (s, u)=\Delta\left(\sum_{j=1}^{5} w_{j} \Delta^{-1}\left(s_{j}, u_{j}\right)\right)=\Delta\left(\sum_{j=1}^{5} w_{j}\left(j+u_{j}\right)\right) \\
& =\Delta((0.225 \times 3.330)+(0.199 \times 3.740)+(0.190 \times 4.090)+(0.205 \times 3.750) \\
& +(0.181 \times 3.330)=\Delta(3.039)=\left(s_{3}, 0.039\right)
\end{aligned}
$$

According to comprehensive 2-tuple linguistic information of first and second grade indicators, a mine that is managed according to the occupational health and safety management system achieves a grade of 'good' economical efficiency, effectiveness, and social efficiency of employee satisfaction with a grade of 'normal' environmental efficiency and individual development. However, the overall employee satisfaction of the OHSAS 18001 has a 'normal' grade, which still needs to be further improved.

\section{Discussion and Suggestion}

Compared with the balance integral card, structural equation model, grey system model, and so on, an improved AHP method ensures reliability of the indicator weight. Furthermore, 2-tuple linguistic information is the result of language evaluation with $\left(s_{k}, u_{k}\right)$, which represents the deviation between the linguistic information set and the most appropriate linguistic phrase in the pre-defined language information set $S$, and solves the defects of the loss of information and accuracy. At the same time, the reliability, integrity, and authenticity of the information is determined by the judgment ability of senior staff, so appropriate training of the methods is greatly needed.

The Mechanism of Cartier [32] tells us that each problem must be split into several simple parts, which is strongly criticized by ecophilosophy. Ecophilosophy insists on a view considering the world as a whole, giving a new explanation to the world's origin, its subject and object, whole and part, the chief and the subordinate. This is the contribution to modern philosophy, which is also the need of sustainable development. We can conclude from the results that the mine paid more attention to economical efficiency, effectiveness and social efficiency, and ignored the environmental efficiency development, which was contrary to the whole development of ecophilosophy.

Ecophilosophy affirms the entity of people, but it discusses intension of entities from the standing of the whole ecosystem, and regards humans as the subject that regulate and control the ecosystem [33]. It can be concluded from the results that the mine paid less attention to hommization which was contrary to the intension of people in ecophilosophy. Enterprises must establish an ecophilosophy concept of development to maintain the balance between human social economy and natural ecology, and to attach great importance to the interdependence between biological, human, and social systems and their environment.

\section{Conclusions}

Enterprise employee satisfaction evaluation is a kind of subjective judgment, wherein the method of preparation of the scale can easily cause information distortion. Additionally, the integrity of information can not be preserved in the process of language integrating. The 2-tuple linguistic information and operator methods overcome the above shortcomings. Five indicators of OHSAS18001 employee satisfaction of individual development, effectiveness, economical efficiency, social efficiency, and environmental efficiency are given and combined with the characteristics of OHSAS18001. Th method of improved AHP is used to calculate the weight of the first and second grade indicators, whose consistency is verified by the root mean square method. The reliability of the results is guaranteed. At the same time, the feasibility of the method is authenticated by the case study of 2-tuple linguistic information, which provides an important reference for the effective operation and 
strategic management of the mine OHSAS18001 and for the coordination and sustainable development of human, economic, social, resource, and environmental protection.

According to the concept of ecophilosophy and OHSAS18001 standard terms, the mine should invest more in the individual development of employees. Giving them more training and development opportunities can not only improve their skills, but it also creates more economical efficiency. At the same time, the mine should strictly implement standards terms of 4.4.6 [16] to establish and maintain documented procedures to cover situations where their irrational environmental behavior could lead to deviations from the OHSAS18001 policy and the objectives, to establish and maintain procedures related to the identified OHSAS18001 environmental risks of goods, equipment, and services purchased and used by the organization, to communicate relevant procedures and requirements to suppliers and contractors, and to establish and maintain procedures for the design of workplace, process, installations, machinery, operating procedures, and work organizations, including their adaptation to human capabilities, in order to eliminate or reduce OHSAS18001 environmental risks at their source.

The geographical position, management mode, and single- or multi-system of different mines in China vary greatly. There are many different ways to evaluate the employee satisfaction of OHSAS18001. Thus, this method allows for necessary adjustments according to the actual situation and experience. For future work, the research in this area should be strengthened, the constructed model should be optimized, and in the selection of evaluation methods, we should consider effectively integrating 2-tuple linguistic information, projection operator, and geometric weighted averaging operator to further enrich the model of employee satisfaction based on the 2-tuple linguistic information.

Acknowledgments: We thank the employees of the case study area for their cooperation in the study and the leaders of the mine for their guidance. The Research Center for Environment and Health of Zhongnan University of Economics and Law in China funded the work reported in this paper.

Author Contributions: All authors contributed to design, method, and analysis reported in the paper. Jan Johansson and Jingdong Zhang conducted the fieldwork, collected and processed the data reported in the paper. Jiangdong Bao and Lena Abrahamsson conducted the analysis and led the writing of the manuscript. Jiangdong Bao developed and prepared all tables and figures. All authors commented on the manuscript, providing insights used in the analysis and discussion.

Conflicts of Interest: The authors declare no conflict of interest.

\section{References}

1. Tian, S.S. Evaluation research of employee satisfactory degree based on two-tuple semantic meaning. J. Henan Inst. Sci. Technol. 2010, 9, 12-16.

2. Delgado, M.; Verdegay, J.L.; Vila, M.A. Linguistic decision-making models. Int. J. Intell. Syst. 1992, 7, 479-492. [CrossRef]

3. Delgado, M.; Verdegay, J.L.; Vila, M.A. A model for linguistic partial information in decision-making problems. Int. J. Intell. Syst. 1994, 9, 365-378. [CrossRef]

4. Delgado, M.; Verdegay, J.L.; Vila, M.A. On aggregation operations of linguistic labels. Int. J. Intell. Syst. 1993, 8, 351-370. [CrossRef]

5. Carlsson, C.; Fullér, R. Benchmarking in linguistic importance weighted aggregations. Fuzzy Sets Syst. 2000, 114, 35-41. [CrossRef]

6. Herrera, F.; Martínez, L. A 2-tuple fuzzy linguistic representation model for computing with words. IEEE Trans. Fuzzy Syst. 2000, 8, 746-752.

7. Du, Q. Benefit Analysis of Safety Investment in Coal Mine Based on DEA; Kunming University of Science and Technology: Kunming, China, 2009.

8. Extremera, N.; Rey, L. Ability emotional intelligence and life satisfaction: Positive and negative affect as mediators. Person. Individ. Differ. 2016, 102, 98-101. [CrossRef]

9. Baptista, R.C.; Paiva, L.A.; Gonçalves, R.F.; Oliveira, L.M.; Pereira, M.F.; Martins, J.C. Satisfaction and gains perceived by nursing students with medium and high-fidelity simulation: A randomized controlled trial. Nurse Educ. Today 2016, 46, 127-132. [CrossRef] [PubMed] 
10. Stathopoulou, A.; Balabanis, G. The effects of loyalty programs on customer satisfaction, trust, and loyalty toward high- and low-end fashion retailers. J. Bus. Res. 2016, 69, 5801-5808. [CrossRef]

11. Agyeiwaah, E.; Adongo, R.; Dimache, A.; Wondirad, A. Make a customer, not a sale: Tourist satisfaction in Hong Kong. Tour. Manag. 2016, 57, 68-79. [CrossRef]

12. Malinen, O.; Savolainen, H. The effect of perceived school climate and teacher efficacy in behavior management on job satisfaction and burnout: A longitudinal study. Teach. Teach. Educ. 2016, 60, 144-152. [CrossRef]

13. Anderson, E.W.; Fornell, C.; Lehmann, D.R. Customer satisfaction, market share, and profitability: Findings from Sweden. J. Mark. 1994, 58, 53-66. [CrossRef]

14. Zehrer, A.; Raich, F. The impact of perceived crowding on customer satisfaction. J. Hosp. Tour. Manag. 2016, 29, 88-98. [CrossRef]

15. Oliver, R.L. Measurement and evaluation of satisfaction processes in retail settings. J. Retail. 1981, 57, $25-48$.

16. General Administration of Quality Supervision, Inspection and Quarantine of People's Republic of China. National Standards of the People's Republic of China, GB/T28001-2011 Occupational Health and Safety Management Systems Requirements. Available online: http://wenku.baidu.com/link?url=91fpXA_ DrfdnyDXePMFpo32DLb9OtkMPtkdzRBLXHLk_5fHTdv0oxp10vsxB5gzNR1owWzwS35O2M5Q_Hh0_ rziuhTeL5FoaBkS1SVeOsCC (accessed on 16 January 2017). (In Chinese)

17. Thomas, L.S. Making and validating complex decisions with the AHP/ANP. J. Syst. Sci. Syst. Eng. 2005, 14, $1-36$.

18. Azadeh, A.; Zadeh, S.A. An integrated fuzzy analytic hierarchy process and fuzzy multiple-criteria decision-making simulation approach for maintenance policy selection. Simulation 2016, 1, 3-18. [CrossRef]

19. Guo, S.J.; Hua, Y. AHP on the basis of index number scale and its usage. J. Nantong Inst. Technol. (Nat. Sci.) 2004, 3, 4-7.

20. Wu, D.T.; Li, D.F. Shortcomings of analytical hierarchy process and the path to improve the method. J. Beijing Normal Univ. (Nat. Sci.) 2004, 12, 264-267.

21. Wei, C.P.; Zhang, Z.M. An algorithm to improve the consistency of a comparison matrix. Syst. Eng. Theory Pract. 2000, 20, 62.

22. Wang, Y.M. An overview of priority methods of comparison matrix. J. Manag. Sci. China 1995, 5, 101.

23. Bao, J.D.; Zhang, J.D.; Li, F.; Liu, C.Y.; Shi, S.P. Social benefits of the mine occupational health and safety management systems of mines in China and Sweden based on a fuzzy analytic hierarchy process: A comparative study. J. Intell.Fuzzy Syst. 2016, 31, 3113-3120. [CrossRef]

24. Chen, S.; Yang, G.F. Eco-environmental quality appraisal of yellow river delta wetland using the improved AHP method. South-to-North Water Divers. Water Sci. Technol. 2011, 9, 99-101. [CrossRef]

25. Bao, J.D.; Johansson, J.; Zhang, J.D. Occupational Disease Assessment of Mine Industry Occupational Health and Safety Management System based on FMEA and Improved AHP Model. Sustainability. 2017, 9, 94. [CrossRef]

26. Le Nguyen, T.; Quyen, H.A.; Nguyen, N.A. Application of fuzzy-analytic hierarchy process algorithm and fuzzy load profile for load shedding in power systems. Int. J. Electr. Power Energy Syst. 2016, 77, 178-184.

27. Chaudhary, P.; Chhetri, S.K.; Joshi, K.M.; Shrestha, B.M.; Kayastha, P. Application of an Analytic Hierarchy Process (AHP) in the GIS interface for suitable fire site selection: A case study from Kathmandu Metropolitan City, Nepal. Socio -Econ. Plan. Sci. 2016, 53, 60-71. [CrossRef]

28. Song, Z.H.; Ma, Q.M. The application of Excel in establishing evaluation index system based on AHP method. Stat. Decis. 2012, 21, 104-105.

29. Herrera, F.; Martinez, L. A model based on linguistic 2-tuples for dealing with multi-granularity hierarchical linguistic contexts in multi-expert decision-making. IEEE Trans. Syst. Man Cybern. 2001, 31, 227-234. [CrossRef] [PubMed]

30. Herrera, F.; Herrera-Viedma, E. Aggregation operators for linguistic weighted information. IEEE Trans. Syst. Man Cybern. Part A Syst. 1997, 27, 646-656. [CrossRef]

31. Wang, X.R.; Fan, Z.P. A method for group decision making problems with different forms of preference information. J.Northeast. Univ. (Nat. Sci.) 2003, 24, 178-181. 
32. Mechanism. Catholic Encyclopedia. 1913. Available online: http://www.newadvent.org/cathen/10100a. htm (accessed on 16 January 2017).

33. Chen, H. Ecological philosophy: A new Philosophical View of Ecological Existentialism. Hebei Acad. J. 2005, $6,113-119$.

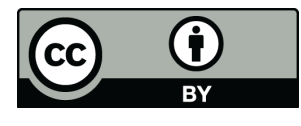

(C) 2017 by the authors; licensee MDPI, Basel, Switzerland. This article is an open access article distributed under the terms and conditions of the Creative Commons Attribution (CC-BY) license (http://creativecommons.org/licenses/by/4.0/). 